\title{
Chapter 3 \\ Providing Universal Access While Avoiding Antiretroviral Resistance: Ethical Tensions in HIV Treatment
}

\author{
Bridget Haire
}

\begin{abstract}
The provision of effective antiretroviral therapy is an ethical imperative, and global access to antiretroviral drugs is an important aspect of this. The other less recognised aspect of effective HIV management is in ensuring that HIV does not become resistant to the drugs used in treatment (and increasingly also in prevention), as multi-drug resistant HIV poses a major threat to the sustainability of current responses to HIV control. In resource-constrained environments, the rapid scale up of access to life-saving anti-HIV treatment was achieved using a public health approach that standardised antiretroviral regimens, minimised laboratory monitoring, and devolved responsibilities from clinicians where necessary. In recent years demand for antiretroviral treatment has increased due to new understandings of the clinical importance of early treatment, but global investment has declined. Exponential growth of the population using antiretrovirals without careful monitoring increases the risk of significant antiretroviral drug resistance. In this chapter, I consider the example of single-drug interventions to prevent parent-to-child HIV transmission, and how the implementation of that strategy increased health risks for mothers. I argue that while global antiretroviral scale up must continue, laboratory monitoring at individual and national levels needs to improve to maintain treatment effectiveness, and protocols for moving people from failing regimens need to be strengthened.
\end{abstract}

Keywords Ethics · Public health - Infectious diseases

B. Haire $(\square)$

Kirby Institute, University of NSW, Sydney, Australia

e-mail: b.haire@unsw.edu.au 


\subsection{Introduction}

With an estimated 36.9 million people living with HIV worldwide (WHO 2018), the provision of effective antiretroviral therapy (ART) is an ethical imperative. Ensuring that people with HIV can access ART is an important part of this, but another less recognised aspect is ensuring that the HIV does not become resistant to the drugs used in the treatment, as multi-drug resistant HIV poses a major threat to the sustainability of current responses to HIV control. There are, however, ethical tensions about striking the right balance between maximising ART access and minimising the risk of the emergence of drug resistant HIV. In resource-constrained environments these tensions are concerned with relative investment in drugs as compared with laboratory monitoring, and sometimes 'blaming' discourses regarding nonadherent people with HIV.

In 1996 it was established that antiretroviral drugs used in combination could control HIV replication ('viral load') in individuals and thus prevent HIV from destroying the CD4 cells that protect against infection (Arts and Hazuda 2012). Over time, the control of viral replication was shown to prevent people with HIV from becoming immune suppressed and developing the opportunistic infections associated with acquired immune deficiency syndrome (AIDS) (Moore and Chaisson 1999). ART could also reverse immune damage, to some extent (Kaufmann et al. 2005). Thus, combination ART could prevent AIDS and prolong, and perhaps save, the lives of people with HIV. Initial drug regimens however were often highly complex. The drugs were associated with serious side effects and were prohibitively expensive, as were the laboratory tests used to monitor both the impact of the drugs on viral load (viral load tests) and the degree of immune functionality (measured by CD4 cell tests). Hence there were significant barriers to access to these regimens for the majority of people living with HIV.

The stark injustice of life saving medication being inaccessible to the majority of those living with HIV, concentrated predominantly in low and middle income countries (LMIC) and especially in sub-Saharan Africa, was the catalyst for a worldwide treatment access movement that put pressure on governments, drug companies and non-government organisations to find solutions to make ART access more equitable. In response to the treatment access movement the World Health Organization launched its first 'public health' program for HIV treatment, an approach that aimed for universal access, starting with the initial goal of getting 3 million people onto ART by 2005, the ' 3 by 5 Programme' (WHO 2003a, b). This approach was supported and facilitated by the creation of two major donor organisations - The Global Fund for HIV, TB and Malaria (the Global Fund), and the (US) President's Emergency Program for AIDS Relief (PEPFAR) - that supplied drugs to LMIC (Smart 2006). Even with these donors, it was not feasible to mimic the highly individualised approach to ART prescription in affluent countries that relied heavily on expensive and resource-intensive laboratory monitoring. Accordingly, the program standardised both ART regimens (the drugs) and monitoring (including tests that measure the effect of the drugs on viral load and those that measured the impact of 
HIV on the immune system). In some LMIC, nurse-practitioners performed roles usually reserved for doctors. Thus, the 'public health' approach was designed to facilitate ART delivery in resource-poor settings with limited health infrastructure, simplifying, standardising and devolving responsibilities as required (WHO 2003a, b). (Note, while the ' 3 by 5 Programme' was the initial iteration of this approach, the WHO has continued to champion universal access programmatically using a public health approach. For simplicity's sake, in this chapter I will treat the WHO universal access/public health approach as a strategy that started with ' 3 by 5 ' and has continued to this day, albeit with various adjustments and shifts in nomenclature.)

This chapter will first describe the mechanism for the development of HIV drug resistance and detail elements of the 'public health' approach to ART. It will then discuss how and why the 'public health' approach to ART in lower income countries was a reasonable trade-off that enabled widespread access to HIV treatment, despite increasing the likelihood of the development of drug resistance. In the medium to long term, however, strategies to avoid, contain and diagnose drug resistance will be critical to the sustained management of the epidemic, and to reaching the global goal of virtual elimination of new HIV infections. The tensions between a public health approach that focuses on access and an increasing emphasis on effective use of resources will be explored, using the history of mother-to-child prevention programs as a case study. The increasing use of ART-based prevention strategies for sexual transmission will also be considered. Finally consideration will be given to issues likely to impact on responses to drug resistant HIV in a context where guidelines recommend universal treatment, but access to uninterrupted drug supplies and high quality monitoring standards are limited.

\subsection{Drug Resistant HIV}

HIV is a highly mutable virus, meaning that as it replicates it makes errors and hence changes and evolves over time within the human host. These changes are random, though the principles of natural selection mean that over time and in the absence of treatment, changes that make the virus more replication competent will crowd out those that make it less fit.

Drug resistant HIV develops when a person is taking ART that is insufficient to fully suppress viral replication. This creates the conditions of selective pressure, so that viral copies that are less sensitive to the drugs have a competitive advantage over other viral copies, and begin to push the evolution of HIV in the particular body to become increasingly resistant. If the ART regimen is sufficient to fully suppress replication, this will not occur and hence resistance will not develop. Standard ART regimens will fully suppress viral replication if adherence is good, if no biological event disrupts individual drug absorption and metabolism, and unless the person was infected with a strain of HIV that had already evolved to be resistant to some or all of the drugs in the particular ART regimen. 
In terms of the potential development of ART resistance, all ART drugs are not equal - the strength of antiretroviral action can differ, and some drugs are more vulnerable to the development of resistance. ART drugs work by targeting and blocking selected enzymes or proteins of HIV that enable it to replicate and infect cells. Changes to particular HIV regions that the ART drugs target make the drugs less effective. Some regions of HIV are more prone to mutation than others, and some drugs lose their effectiveness in the face of HIV evolution faster than others. For example, a single mutation reduces susceptibility to the drug nevirapine (this is called having a low genetic barrier to resistance) (Luber 2005). Drug resistance is also not a black and while phenomenon, in that a particular mutation or set of mutations may diminish susceptibility to particular drugs, but not completely stop working in terms of controlling HIV replication. For example, a person taking ART who has a detectable but low viral load may have some drug resistance, but may also be receiving clinical benefit from the drugs.

ART drugs are classified into classes that are determined by where and how they target HIV replication. While ART drugs within the designated classes work in slightly different ways, developing resistance to one drug in a class can also confer either resistance (or at least reduced sensitivity) to other drugs in the class. Accordingly, if a person has to change from one ART regimen to another, the second regimen needs to comprise drugs to which there is no likely pre-existing cross resistance to maximise the likely success. Further, in high income countries like Australia, when a person is first diagnosed with HIV, genetic resistance testing determines whether the person has been infected with a resistant strain of HIV that is less likely to respond optimally to standard treatment (if resistance is found, different regimens are used).

In summary, there are four main causes of drug resistance: acquiring a drug resistant strain; being prescribed a regimen that is incompletely suppressive (usually single or dual therapy combinations, or a triple combination that is too weak); non-adherence (failing to take pills as prescribed and on time); and taking drugs intermittently (where fluctuating blood levels of medication allow HIV replication under selective pressure).

\subsection{A Word on HIV Monitoring}

There are two highly significant tests used to monitor HIV disease progression and the effectiveness of ART in an individual: Tests that count CD4 cells per cubic ml of blood, and viral load tests. CD4 cells are immune cells that are targeted by HIV. A healthy person would have a CD4 cell count in excess of 500. Once a person's CD4 cell count drops to 200 or below s/he is at serious, imminent risk of HIV-related opportunistic infections. A person with HIV who has a CD4 cell count above 200 but below 500 is showing some HIV-related damage. There is a strong relationship between declines in CD4 cell count and disease progression, and in CD4 cell 
recovery and effective ART treatment (Mellors et al. 1997). CD4 counts are thus a useful tool in the management of HIV disease.

Viral load tests measure viral RNA in the peripheral blood. The higher the viral RNA number is, the greater the level of viral replication, and the greater the risk of disease progression (Mellors et al. 1996). 'Undetectable viral load' means that viral replication is suppressed below the level of detection on the test used (tests have become much more sensitive since they were first available). An undetectable viral load is the individual goal of ART treatment. At the strategic level, having $90 \%$ of people who know their HIV status on ART, and $90 \%$ of those with an undetectable viral load is a current global goal (UNAIDS 2017).

\subsection{Key Elements of the 'Public Health' Approach to HIV}

To maximise access to life-saving ART, WHO prioritised getting ART drugs into the bodies of people with HIV, and maximising the capacity to deliver ART by processes of standardisation, simplification and, where necessary, syndromic management rather than laboratory monitoring. The core underlying principles were identified as urgency, equity and sustainability (Macklin 2004).

Standardised first line and second line ART regimens were a critical aspect of the approach. In determining the drugs used in these regimens, a balance had to be struck between user-friendliness (pill burden, dosing routine, side effect profile), effectiveness (probability of viral load suppression), and cost (WHO 2003a). Further, the second line regimen had to be effective against the ART resistance likely to develop (for whatever reason) to the drugs in the first line regimen. (Third line regimens are also detailed in guidelines.) These standardised regimens were presented as algorithms that were flexible enough to allow for the different availability of certain drugs in particular countries due to purchasing arrangements, and to allow some substitutions for people who had different health needs, such as those who were pregnant, had other infections like TB or viral hepatitis. The standard algorithms for first and second line treatment have changed since the first iteration of the WHO universal access program, as further information about drug efficacy emerged (for example, a first line regimen recommended in 2003 was found to be insufficient of suppress very high viral loads found in people initiating treatment in advanced disease), and alternative drugs became available to replace ART associated with significant toxicities (Gulick et al. 2004). Both inadequate viral suppression and high levels of side effects are significant for the development of resistance - incomplete suppression create the conditions for the emergence of resistance, and ART toxicities can reduce adherence, also potentially resulting in incomplete suppression.

The issue of how to prioritise people for treatment access was another critical element of the approach. Utility, equity and concern for the worst-off (the principle of maximin) were key considerations (Macklin 2004). Initial WHO guidance for resource constrained settings recommended that ART should be commenced in 
those at high and proximate risk of clinical disease progression and/or death (those diagnosed with Stage IV HIV disease regardless of CD4 cell count; those with CD4 cell counts below 200 regardless of disease staging; those with stage III HIV disease and CD4 counts below 35) (WHO 2003a). Targeting ART at those at experiencing or at imminent risk of serious HIV disease was acceptable in a moral sense as it prioritised treatment for those with greatest clinical need, and it also greatly reduced the pool of people eligible for treatment, as only a fraction of those living with HIV would require treatment under such guidance. The downside to this approach was that many people tested and found HIV positive would not be eligible for treatment, increasing the potential of significant loss to follow-up (Rosen and Fox 2011). It is important to note that while WHO guidelines play an important normative role in shaping standards, they are in no sense binding, and the expectation of their use is that countries draw from them and adapt them according to relevant contextual factors (WHOF 2006).

Laboratory monitoring was simplified in WHO guidance documents, with CD4 cell tests recommended but not mandatory both at baseline and for subsequent monitoring of treatment efficacy at 6-12 month intervals. Haemoglobin testing would occur depending on the drug regimen (it was required if AZT was included.) Other blood work such as white blood cell and liver function tests were to be ordered as determined by clinical symptoms, and viral load testing - the gold standard for measuring ART response - not even mentioned. Developing guidelines for the surveillance of drug resistance was however one of the itemised strategic actions of the initial ' 3 by 5 Programme' (WHO 2003a, b). The low level of laboratory monitoring was contentious, given that the longer a person stays on a failing regimen, the greater the opportunity both for the development of resistance and the loss of clinical benefit. Accordingly, a randomised control trial (RCT) investigated whether (and to what extent) ART could be delivered safely without laboratory monitoring (the DART study). This study, first reported in 2009 and published in 2010, found that differences in outcomes occurred after the second year of treatment. Investigators argued that this justified only introducing CD4 cell tests at 24 months, and prioritising spending on ART drugs rather than resource-intensive monitoring (DART Trial Team 2010). Of note, this study only looked at clinical outcomes, in people who already had advanced HIV disease, not at the drug resistance outcomes.

\subsection{Changes in Eligibility for ART}

At the inception of the global universal access program, there was no clear scientific evidence as to the best time to commence ART, except that it clearly prolonged life for those who commenced with low CD4 counts (below 200). From the mid 1990s, many argued on the basis of modelling from other infectious diseases that HIV replication should be suppressed as early and as completely as possible. 'Hit hard, hit early', was the catch cry (Ho 1995). Mitigating against this was the fact that serious HIV disease did not usually occur until 6-10 years after infection, and the 
problem that ART regimens in the mid to late 1990s were hard to take (with high pill burdens, specific food and or fasting requirements for dosing particular drugs, and high rates of side effects). Of course the rapacious cost of ART was also significant - for example in 1997, 6 months use of the drug AZT was costed at \$US 800 it is now an estimated \$US 237 (Pharmacychecker 2017).

From the late 90s, a series of RCTs clarified issues pertaining to treatment commencement, indicating that better outcomes were achieved with earlier treatment initiation. These included CIPRA-HT001, a study in Haiti that showed that people who started ART with CD4 counts higher than 200 had a significantly reduced risk of death (Severe et al. 2010). This result was underscored by findings from the SMART Study, which was designed to test whether structured ART treatment interruptions were safe (people with HIV took treatment interruptions or 'holidays' for a variety of reasons, including relief from side effects). The SMART Study found that not only did treatment interruptions increase the risk of disease progression at any CD4 level, but that the greater time spent with a CD4 count below 350, the greater the risk of HIV disease progression and death (Strategies for Management of Antiretroviral Therapy Study Group 2008). These findings together were the catalyst for a revision of WHO guidelines for resource-constrained environments to recommend ART commencement at the higher CD4 level of 350 (WHO 2009). Two further studies have been critical in pushing WHO ART guidelines toward recommending treatment at higher levels - the HPTN 052 Study, which showed that early ART treatment reduced onward HIV transmission to sexual partners by $96 \%$ (Cohen et al. 2011), and then the START Study, reported in 2015, which showed a clear clinical benefit from early treatment initiation (at CD4 levels of 500 and above), with people who initiated treatment immediately showing significant benefit compared with those who did not initiate ART until their CD4 counts had dropped to 350 or below (The Insight START Study Group 2015). This finding resulted in a revision of WHO guidelines, such that immediate ART therapy is now recommended for any person diagnosed with HIV, regardless of CD4 cell count.

This change to WHO ART guidelines is based in robust evidence about the beneficial impact of immediate ART on health, and where taken up in national guidelines, may reduce the numbers of people diagnosed with HIV who get lost to follow -up due to being deemed ineligible for immediate ART. It also has some problematic implications. Firstly, it increases exponentially the estimates of people living with HIV who require ART, which may increase the likelihood of rationing taking place where supplies are inadequate. Secondly, notwithstanding the fact that some resource-poor countries may continue to align their national guidelines to earlier iterations of the WHO guidance, the expansion of eligibility for ART puts greater demand on ART stocks, and increases the risks of stock-outs, which could have an impact on the development of drug resistance. Even if drug supplies were perfect, human adherence will not be, so the logical result of millions more people taking lifelong ART over many decades must be an increase in drug resistance. Thirdly, increasing the number of people eligible for treatment may increase pressure at country level to continue to prioritise wider access to ART drugs at the expense of improving monitoring, exponentially expanding the risk that large scale poorly 
monitored ART use poses with regard to development and transmission of drug resistant ART (at higher CD4 cell counts, the risk of death is far more remote that at lower one, so the risks of drug resistance are not so obviously balanced by the overwhelming benefit of prevention of illness and death). Fourthly, there is a major paradigm shift in terms of health communication in shifting ART treatment from something required for people with ill or declining health status to treating people who are well, and requiring this treatment to be maintained over a lifespan. Thus, well-targeted health promotion, innovative and effective adherence support, improved monitoring to detect signs of resistance early, and affordable, tolerable second, third and fourth line (or rescue) regimens all need to be integrated into this ambitious expansion of ART eligibility.

\subsection{ART in Pregnancy in LMIC: A Case Study}

"Once started, antiretroviral therapy is for life...." states the introduction to the ' 3 by 5 Programme' (WHO 2003a, b). The reason that ART treatment, once started, is to be continued for life is to maximise health by maintaining a supressed HIV viral load, and preventing the development of drug resistance that could occur if ART is started and stopped (as the various drugs in combination ART regimens have different half-lives, it is complex to stop a regimen in a way that ensures that there is no lingering, suboptimal antiviral activity that could provide the conditions for drug resistance to develop). A brief look at the history of interventions aimed at preventing vertical infection (from mother-to-child), however, shows that the maxim of ART therapy being for life has frequently not been the case with regard to provisions of ART to pregnant women. The case study below, I suggest, is an example of how the prevention benefit of ART was initially prioritised above the healthcare of pregnant women with HIV.

The prevention of HIV transmission from mother to child (PMTCT) was the first instance of effective biomedical HIV prevention, achieved in 1994 by the ACTG 076 study (Connor et al. 1994). Famously, this breakthrough spawned a series of placebo-controlled trials in LMIC using less intensive, cheaper regimens (see Wade et al. 1998). This chapter will not delve into the ethical quagmire surrounding the use of placebos despite an effective preventative intervention having been established, as this has been exhaustively covered elsewhere (see Macklin 2001). Instead, this chapter will recount and analyse what occurred programmatically in PMTCT following the results of the HIVNET 012 Trial, which established that a single dose of the ART drug nevirapine to both mother and infant reduced transmission in the first $14-16$ weeks of life by nearly $50 \%$ in a breastfeeding population (Guay et al. 1999). This regimen, while not as effective as the ACTG 076 regimen, was considered to be a major breakthrough for LMIC. Unlike ACTG 076, it did not require Caesarian section, it did not require treatment uptake in mid pregnancy, it did not require intravenous ART during delivery, and it appeared to work in breastfeeding populations. All of these factors made it significantly more feasible to implement at 
scale in LMIC. (Of note, when the Food and Drug Administration looked at the data from this trial, it found serious anomalies in the conduct of the trial. Accordingly questions remain regarding the validity of this study's results (Cohen 2004; Institute of Medicine Committee 2005).

One of the issues with the early WHO guidelines that recommended ART treatment only for people with signs of immune damage and/or HIV disease was the problem it created regarding pregnant women with HIV. Despite pregnant women in high income countries achieving excellent health and prevention outcomes with early ART, it meant that pregnant women in LMIC without clinical signs of HIV disease and with $\mathrm{CD} 4$ counts above levels recommended for treatment were deemed not to require ART for their own health, despite knowledge that ART would reduce transmission to infants. This created an artificial distinction between the treatment use of ART and prophylactic use in pregnant women - prophylactic use only was recommended. In other words, a pregnant woman with HIV but in otherwise good health would get access to ART to protect her infant, but this would be short-term.

Despite its appeal in terms of feasibility, the nevirapine single-dose ${ }^{1}$ regimen had a very specific problem. Although a potent ART drug, nevirapine as noted earlier has a low genetic barrier to resistance, and a long half-life. Thus the drug persists in the blood stream for a long time after a dose, but at concentrations insufficient to fully suppress viraemia. This creates a good environment for the development of drug resistance, and a single dose is enough for this to occur. Exposing women and their infants to this regimen could thus potentiate the development of drug resistance, which might reduce the efficacy of the intervention for any subsequent pregnancies, and limit treatment options for the mother and potentially for the infant, should the infant be HIV infected despite the use of nevirapine prophylaxis.

The publication of the long-term results HIVNET 012 trial was accompanied by a controversial commentary by Karen Palmore Beckerman (2003) that argued that the implementation of single-dose nevirapine would leave 'between $20-100 \%$ of women who received prophylaxis resistant to [the class of drugs to which nevirapine belongs]'. While the claim that up to $100 \%$ would develop resistance was hyperbolic (Wilfert 2003), the issues she raised were prescient. Beckerman argued that pregnant women with HIV should be treated with effective ART regimens both for their own sake and to enable their survival - citing the devastating example that three women who participated in HIVNET 012 died of AIDS within 56 days of delivery. She suggested that adding a combination ART 'tail' to the nevirapine regimen could reduce the likelihood of resistance (a suggestion later taken up in WHO guidelines in 2010), and that the primary focus be on sustained and sustainable ART treatment.

Beckerman's (2003) article built on an earlier commentary of hers (Beckerman 2002) where - again in response to the publication of short-course trial to reduce mother-to-child transmission - she argued that the risks of programs using single or

\footnotetext{
${ }^{1}$ This regimen is referred to in the literature as 'single dose' though it in fact involves two doses one to mother and one to infant. For simplicity's sake I'm adopting the common usage.
} 
dual ART drugs were increasing as the prospect of good treatment access in LMIC grew, given that women who had used short-course ART would be more likely to experience treatment failure due to drug resistance. With limited treatment options available, failing first line ART options would increase the risk of the mother dying, which would in turn increase risk for surviving children, Beckerman argued.

There is a considerable literature on the clinical implications of nevirapine resistance, with much focus on whether or not it 'fades' over time such that subsequent introduction of an ART regimen containing nevirapine might still have clinical utility as either treatment or prophylaxis (Johnson et al. 2005; Lockman et al. 2007, McConnell et al. 2007; Stringer et al. 2010). Regardless of whether or not resistance 'fades' to a level that would result in nevirapine-based combination being effective in women exposed to single-dose PMTCT, it is clear that, as others have noted, the prevention of transmission to infants took precedence over the health security of women with HIV, at least for a time when these single-dose nevirapine programs were widespread (Eyakuze et al. 2008). As noted in a meta-analysis, these programs ultimately resulted in a high burden of drug resistance in women and children, with significant potential to contribute to increase failure of first line ART therapy (Arrivé et al. 2007; Samuel et al. 2016).

From 2003, WHO guidance recognised that pregnant women should not be prescribed ART regimens likely to result in drug resistance, and had specifically provided a warning about the single-dose nevirapine regimen. By 2012 the policy of women cycling on and off ART with successive pregnancies was superseded by 'Option $\mathrm{B}+{ }^{2}-$ a recommendation that pregnant women with HIV should be prescribed fully suppressive ART regimens, and kept on them for life, regardless of CD4 cell count or HIV disease stage. While Option B+ has not been fully implemented in all LMIC, it is the only PMTCT option that fully applies the 'rules' of effective ART treatment - that regimens should be fully suppressive, and taken for life. Ironically, there have been reports of significant loss to follow up with these programs, perhaps because women with HIV had being educated to accept intermittent therapy unless they themselves were in poor health (Tenthani et al. 2014)

Reflecting on the history of PMTCT, there are tensions between the prevention imperative, emerging knowledge about optimal timing of ART, the status of women's health, equity, and the constraints of the early stages of implementation of the morally praiseworthy but hugely ambitious goal of universal access. In the late $90 \mathrm{~s}$ and early 2000s, the nevirapine single-dose regimen had appeal in terms of its simplicity, its low drug burden, and the fact that it could be implemented during

\footnotetext{
${ }^{2}$ Option B+ was added to two previous options, option A and Option B. Briefly, Under Option A, women received ARV prophylaxis prenatally and during delivery, along with an antiretroviral postpartum "tail" regimen to reduce risk of drug resistance, and their infants received postpartum antiretroviral prophylaxis throughout the duration of breastfeeding. With Option B, all pregnant and lactating women with HIV were offered ARV - beginning in the antenatal period and continuing throughout the duration of breastfeeding. At the end of breastfeeding those women deemed to not yet require ARV for their own health would discontinue the prophylaxis and continue to monitor their CD4 count, eventually re-starting ARV when their CD4 cell count fell below 350 cells.
} 
delivery. In short, it had been shown to be better than nothing at a time when 'nothing' was still seen in some quarters as an acceptable comparator. While the shift away from the single dose regimen happened relatively quickly (at least in terms of normative guidance, if not in practice), its impacts are likely to live on in the form of suboptimal response to first line ART regimens and transmitted drug resistance (Kébé et al. 2014).

It took 6 years from the initial reporting of the SMART study - which showed that starting and stopping ART was detrimental - for PMTCT guidance to recommend life-long treatment for women who initiated ART due to pregnancy. On the one hand, this seems shocking. On the other hand, due to PMTCT programs even with their limitations, women in LMIC tended to access ART earlier than men, and hence have had lower risk of death from HIV disease than men (Beckham et al. 2016; Taylor-Smith et al. 2010), so further strengthening care access for women may have seemed inequitable in some contexts. It could be argued that the history of PMTCT in LMIC demonstrates a programmatic emphasis on prevention without adequate regard for the potential development of drug resistance, and subsequent drug failure in mothers and pregnant women with HIV. It is probably more reasonable, however to say that the complexity of implementing best practice PMTCT in the midst of programmatic scale up of ART access in a context where knowledge about ART changed significantly made compromise and incremental steps forward hard to avoid, if not inevitable. Nevertheless, exposure to sub-optimal therapy through PMCTC programs has left a legacy of drug resistance in some settings (Rowley et al. 2016; Cambiano et al. 2013).

Now that lifelong ART from the point of diagnosis is the WHO recommendation for everyone, exponentially more people will be accessing ART prior to any clinical indications of HIV. That number will be expected to keep increasing, as ART access should enable people with HIV to live normal lifespans. In this context the risk of the development of drug resistance must necessarily increase, given the difficulties of maintaining good-enough adherence for large populations over long periods of time. While the low-level clinical monitoring approach to HIV disease had an important role in enabling the 'public health' roll out of ART in LMIC, this approach would not be sustainable in populations accessing treatment much earlier in HIV disease.

\subsection{Addressing ART Resistance}

Addressing the risk of drug resistance requires strategic action at many levels. Action is required at the community and local levels, to develop and sustain programs that support individual adherence and that work to address systemic problems that can affect adherence (by improving systems of clinic appointments and transport and drug access, for example). Scaling up monitoring and investing in viral load testing will become critical so that people who are on failing regimens for whatever reason - are identified quickly and either supported to improve adherence or switched to second line therapy. Development of viral load and CD4 testing 
technologies that are better adapted to use in remote and/or resource constrained settings, and which do not require highly trained laboratory staff, should obviously be prioritised to reduce the dependence of remote and under-resourced clinics on distant laboratory services. Research and development into new effective ART that are well tolerated and are effective against current common resistance patterns in HIV - and new modes of delivery, such as periodic injectables - are of course highly desirable.

While some commentators have suggested withdrawing or withholding ART from non-adherent people rather than providing practical support and transitioning them as necessary to second line ART, this perspective positions poor adherence as an individual (and moral) issue (Chawana \& Bogaert 2011). A perspective of adherence that is informed by public health and human rights, on the other hand, recognises that while adherence is an individual behaviour, it is highly determined by structural factors and social context, which can and should be modified to make adherence as simple and as socially acceptable and socially desirable as possible. There is evidence that people in certain demographics (such as young people) may have more trouble with adherence (Haire 2015). Withholding or withdrawing ART on the basis of poor adherence from young people, for example, would not only remove the benefits of early treatment from the demographic with the most to gain, it would also remove the prevention benefit from a population group highly likely to be sexually active. Accordingly, it would seriously undermine global targets for universal access. Recognising, working with, and seeking to ameliorate adherence problems in the social groups that experience them, while advocating for ART that provide different delivery options such as periodic injection, makes sense from a public health and human rights perspective. Universal access goals aim for $90 \%$ of people with HIV knowing their status, $90 \%$ of those being on ART and $90 \%$ of those having undetectable viral loads by 2020 (UNAIDS 2017). Using 2017 figures, this would require more than 14 million more people to be on ART within 2 years considerable investment in supportive and responsive programming to achieve, support and sustain adherence must be factored into such ambitious plans.

\subsection{Biomedical Prevention and Drug Resistance}

Biomedical prevention is the use of antiretroviral drugs to prevent HIV transmission. It includes ART use after exposure to prevent transmission (post-exposure prophylaxis or PEP), ART use prior to exposure in HIV negative people at high risk of HIV acquisition to prevent transmission (pre-exposure prophylaxis or PrEP), and ART use in HIV positive people to fully supress HIV replication, preventing onward transmission to sexual/injecting partners ('treatment-as-prevention'). The latter two strategies are highly effective and well supported by both RCT and observational data (data on PEP is less robust as it has not been tested in an RCT). PMTCT programs contain elements of two biomedical approaches: maternal viral load is reduced to reduce infectiousness, like treatment as prevention, and infants receive 
ART to prevent or abort HIV acquisition, similar to PrEP and PEP. The preventative aspect of early ART treatment is one aspect (in addition to the clinical benefit for the person with HIV) that makes implementation of ART programs in people immediately after diagnosis attractive to governments and donors - it has the potential to reduce onward infection. Similarly, while investment in PrEP programs has been slow, there is a steady increase in availability (AVAC n.d.).

In adherent participants, both PrEP and treatment -as -prevention have shown close to $100 \%$ protection in trials (Grant et al. 2014; Anderson et al. 2012; Bavinton et al. 2018; Rodgers et al. 2016, 2018; Cohen et al. 2011). There has however been at least one verified example of HIV transmission occurring in an adherent PrEPtaker, due to being exposed to HIV that was resistant to both the drugs used in the PrEP combination (Knox et al. 2016). With regard to treatment -as -prevention, having a lowered viral pool in a population would be expected to translate into fewer transmissions at population level. To have confidence in treatment -as -prevention at an individual level, however, the sexual partner with HIV needs to be confident that his/her viral load is sustained at an undetectable level, and that requires access to viral load tests at regular intervals. A combination of PrEP and treatment-as-prevention is being trialled in LMIC in serodiscordant couples, where the HIV positive partner is initiated on ART and the negative partner on PrEP, until such time as the positive partner has a sustained undetectable viral load. For this to succeed programmatically, viral load monitoring is required for the ongoing protection of the negative partner (should the positive partner be non-adherent, the negative partner would run the risk of acquiring HIV, and possibly drug resistant HIV), and to maintain the efficacy of the regimen for the positive partner. In this new era of biomedical prevention and all people with HIV being eligible for ART, the need to implement viral load monitoring at scale is increasingly being recognised (WHO 2016).

\subsection{Monitoring Drug Resistance Beyond the Individual}

In high income countries, when people are diagnosed with HIV, a blood sample is sent to a laboratory for genotypic testing - a form of analysis that provides information of whether the person has been infected with a strain of HIV that is resistant (or less susceptible) to particular ART drugs. The person is then prescribed an appropriate regimen that will be effective against the person's HIV. ${ }^{3}$

In LMIC surveillance of HIV drug resistance includes country specific surveys of HIV genotypes to determine incidence of transmitted drug resistance in

\footnotetext{
${ }^{3}$ Genotyping HIV also has some risks in high income countries, such as the potential to use these data to track 'infection trees' - map pathways of infection. This is ethically problematic in terms of maintaining an enabling environment, as it uses information gleaned from people who test positive for HIV intended to maximise their health outcomes for a purpose that could potentially lead to criminalisation.
} 
populations, through a WHO network of designated laboratories (Bertagnolio et al. 2008). In LMIC this information is directed towards making country or region-wide decisions about first line treatment, and other programs such as the drugs used in post-exposure prophylaxis. In addition, early warning indicators provide information about critical aspects of whether/how people are accessing ART. These include on-time pill pickup; retention in care at 12 months following ART initiation; whether pharmacies experience stock-outs; prescribing practices (whether regimens prescribed meet national or international guidance regarding adequate viral suppression); and, in settings where viral load testing is implemented, viral load suppression at 12 months following ART initiation. These early warning indicators are well designed to signal structural problems, like interruptions in drug supply and poor prescriber compliance with guidelines, and access issues such as whether drug collection systems are 'good enough' (reliable and convenient) for people to be able to pick up pills on time. In 2017, WHO reported 'brisk' implementation of these systems, with 26 countries having completed or currently completing surveys, and 14 having reported data (WHO 2017). Of note, in countries or regions where drug stock outs are frequent, the benefit of putting people with high CD4 counts onto ART would need to be weighed against the individual and public health concern of the development of resistance, should ART be repeatedly started and stopped (for people with CD4 counts lowered by HIV, the clinical benefit of the ART is likely to outweigh the risk). Ideally, of course, the stock out issues should be addressed effectively.

\subsection{Conclusion}

Although hope and hype about vaccines and cures for HIV circulate, ART is currently the most effective tool for the elimination of HIV as a public health threat, both in terms of treatment and prevention (in combination with or as an adjunct to condoms). Preventing the emergence of drug resistance at levels that compromises treatment efficacy and ART based prevention is crucial.

With PMTCT programs, the emphasis on the simplest and cheapest regimens, maintained well after the implications for drug resistance were apparent, has already compromised optimal response to ART for too many women and children. This cannot be repeated in the expansion of ART access. Standardised first, second and third line ART has facilitated the rapid scale up of ART in LMIC, and saved many lives. To build on this, a highly strategic approach to monitoring drug resistance in populations and individuals needs to be taken, with viral load monitoring supporting more rapid change to second and third line therapies as required, rather than running the risk of keeping people on failing regimens. Supporting adherence at health service, community, family and individual levels also requires investment - investment in research, to find context-appropriate solutions that work, in communities to provide labour, and in systems to streamline the medical and dispensing processes and practices that can facilitate or hinder access and adherence. While refinements in drug development may make adherence simpler in years to come, the challenge is 
to ensure that programming now is responsive to problem of resistance, and that existing drugs and monitoring are carefully deployed to optimise longevity of tools currently available.

\section{References}

Anderson, P.L., D.V. Glidden, A. Liu, S. Buchbinder, J.R. Lama, J.V. Guanira, et al. 2012. Emtricitabine-tenofovir concentrations and pre-exposure prophylaxis efficacy in men who have sex with men. Science Translational Medicine 4 (151): 151 ra125.

Arrivé, E., M.-L. Newell, D.K. Ekouevi, M.-L. Chaix, R. Thiebaut, B. Masquelier, et al. 2007. Prevalence of resistance to nevirapine in mothers and children after single-dose exposure to prevent vertical transmission of HIV-1: a meta-analysis $\dagger$. International Journal of Epidemiology 36 (5): 1009-1021.

Arts, E.J., and D.J. Hazuda. 2012. HIV-1 antiretroviral drug therapy. Cold Spring Harbor Perspectives in Medicine 2 (4): a007161. https://doi.org/10.1101/cshperspect.a007161.

AVAC. PrEP Watch. Scaling up country updates. http://www.prepwatch.org/scaling-up/ country-updates

Bavinton, B.R., A.N. Pinto, N. Phanuphak, et al. 2018. Viral suppression and HIV transmission in serodiscordant male couples: An international, prospective, observational, cohort study. Lancet. Published online July 16.

Beckerman, K.P. 2002. Mothers, orphans, and prevention of paediatric AIDS. The Lancet 359 (9313): 1168-1169.

- 2003. Long-term findings of HIVNET 012: The next steps. The Lancet 362 (9387): 842-843.

Beckham, S.W., C. Beyrer, P. Luckow, M. Doherty, E.K. Negussie, and S.D. Baral. 2016. Marked sex differences in all-cause mortality on antiretroviral therapy in low- and middle-income countries: A systematic review and meta-analysis. Journal of the International AIDS Society 19 (1): 21106.

Bertagnolio, S., I. Derdelinckx, M. Parker, J. Fitzgibbon, H. Fleury, M. Peeters, et al. 2008. World Health Organization/HIVResNet drug resistance laboratory strategy. Antiviral Therapy 13 (Suppl 2): 49-57.

Cambiano, V., S. Bertagnolio, M.R. Jordan, J.D. Lundgren, and A. Phillips. 2013. Transmission of drug resistant HIV and its potential impact on mortality and treatment outcomes in resourcelimited settings. The Journal of Infectious Diseases 207 (Suppl 2): S57-S62.

Chawana, R., and D.K. van Bogaert. 2011. Risk management in HIV/AIDS: Ethical and economic issues associated with restricting HAART access only to adherent patients. African Journal of AIDS Research 10 (sup1): 369-380.

Cohen, Jon. 2004. Allegations raise fears of backlash against AIDS prevention strategy. Science 306 (5705): 2168-2169. https://doi.org/10.1126/science.306.5705.2168.

Cohen, M.S., Y.Q. Chen, M. McCauley, T. Gamble, M.C. Hosseinipour, N. Kumarasamy, et al. 2011. Prevention of HIV-1 infection with early antiretroviral therapy. The New England Journal of Medicine 365 (6): 493-505.

Connor, E.M., R.S. Sperling, R. Gelber, P. Kiselev, G. Scott, M.J. O’Sullivan, et al. 1994. Reduction of maternal-infant transmission of human immunodeficiency virus type 1 with Zidovudine treatment. The New England Journal of Medicine 331 (18): 1173-1180.

DART Trial Team. 2010. Routine versus clinically driven laboratory monitoring of HIV antiretroviral therapy in Africa (DART): A randomised non-inferiority trial. The Lancet 375 (9709): 123-131. https://doi.org/10.1016/S0140-6736(09)62067-5.

Eyakuze, C., D.A. Jones, A.M. Starrs, and N. Sorkin. 2008. From PMTCT to a more comprehensive aids response for women: A much-needed shift. Developing World Bioethics 8 (1): 33-42. 
Grant, R.M., P.L. Anderson, V. McMahan, A. Liu, K.R. Amico, M. Mehrotra, et al. 2014. Uptake of pre-exposure prophylaxis, sexual practices, and HIV incidence in men and transgender women who have sex with men: A cohort study. The Lancet Infectious Diseases 14 (9): 820-829.

Guay, L.A., P. Musoke, T. Fleming, D. Bagenda, M. Allen, C. Nakabiito, et al. 1999. Intrapartum and neonatal single-dose nevirapine compared with zidovudine for prevention of mother-tochild transmission of HIV-1 in Kampala, Uganda: HIVNET 012 randomised trial. The Lancet 354 (9181): 795-802.

Gulick, Roy M., Heather J. Ribaudo, Cecilia M. Shikuma, Stephanie Lustgarten, Kathleen E. Squires, William A. Meyer III, Edward P. Acosta, Bruce R. Schackman, Christopher D. Pilcher, Robert L. Murphy, William E. Maher, Mallory D. Witt, Richard C. Reichman, Sally Snyder, Karin L. Klingman, and Daniel R. Kuritzkes. 2004. Triple-Nucleoside regimens versus Efavirenz-containing regimens for the initial treatment of HIV-1 infection. New England Journal of Medicine 350 (18): 1850-1861. https://doi.org/10.1056/NEJMoa031772.

Haire, B. 2015. Preexposure prophylaxis-related stigma: Strategies to improve uptake and adherence - A narrative review. HIV/AIDS - Research and Palliative Care 7: 241-249. https://doi. org/10.2147/HIV.S72419.

Ho, David D. 1995. Time to hit HIV, early and hard. New England Journal of Medicine 333 (7): 450-451. https://doi.org/10.1056/nejm199508173330710.

Institute of Medicine (US) Committee on Reviewing the HIVNET 012 Perinatal HIV Prevention Study. 2005. Review of the HIVNET 012 perinatal HIV prevention study. National Academy of Sciences. Washington, DC: National Academies Press.

Johnson, J.A., Li J-f, L. Morris, N. Martinson, G. Gray, J. McIntyre, et al. 2005. Emergence of drug-resistant HIV-1 after Intrapartum administration of single-dose Nevirapine is substantially underestimated. The Journal of Infectious Diseases 192 (1): 16-23.

Kaufmann, G.R., H. Furrer, B. Ledergerber, L. Perrin, M. Opravil, P. Vernazza, M. Cavassini, E. Bernasconi, M. Rickenbach, B. Hirschel, and M. Battegay. 2005. Characteristics, determinants, and clinical relevance of CD4 T cell recovery $<500 \mathrm{Cells} / \mu \mathrm{L}$ in HIV type 1 - Infected individuals receiving potent antiretroviral therapy. Clinical Infectious Diseases 41 (3): 361-372. https://doi.org/10.1086/431484.

Kébé, K., L. Bélec, H.D. Ndiaye, S.B. Gueye, A.A.M. Diouara, S. Ngom, et al. 2014. The case for addressing primary resistance mutations to non-nucleoside reverse transcriptase inhibitors to treat children born from mothers living with HIV in sub-Saharan Africa. Journal of the International AIDS Society 17 (1): 18526.

Knox, D.C., D.H. Tan, P.R. Harrigan, and P.L. Anderson. 2016. HIV-1 infection with multiclass resistance despite Preexposure Prophylaxis (PrEP). 169aLB. In Conference on retro viruses and opportunistic infections, 22-25 February 2016, Boston, Massachusetts.

Lockman, S., R.L. Shapiro, L.M. Smeaton, C. Wester, I. Thior, L. Stevens, F. Chand, J. Makhema, C. Moffat, A. Asmelash, P. Ndase, P. Arimi, E. van Widenfelt, L. Mazhani, V. Novitsky, S. Lagakos, and M. Essex. 2007. Response to antiretroviral therapy after a single, peripartum dose of nevirapine. New England Journal of Medicine 356 (2): 135-147. https://doi. org/10.1056/NEJMoa062876.

Luber, A.D. 2005. Genetic barriers to resistance and impact on clinical response. Medscape General Medicine 7 (3): 69.

Macklin, R. 2001. After Helsinki: Unresolved issues in international research. Kennedy Institute of Ethics Journal 11 (1): 17-36. Project MUSE. https://doi.org/10.1353/ken.2001.0005.

2004. Ethics and equity in access the HIV treatment 3 by 5 initiative: Background paper consultation on ethics and equitable access to treatment and care for HIV/AIDS. http://www. who.int/ethics/background-macklin2.pdf

McConnell, M.S., J.S.A. Stringer, A.P. Kourtis, P.J. Weidle, and S.H. Eshleman. 2007. Use of single-dose nevirapine for the prevention of mother-to-child transmission of HIV-1: Does development of resistance matter? American Journal of Obstetrics \& Gynecology 197 (3): S56-S63. 
Mellors, J.W., C.R. Rinaldo, P. Gupta, R.M. White, J.A. Todd, and L.A. Kingsley. 1996. Prognosis in HIV-1 infection predicted by the quantity of virus in plasma. Science 272 (5265): 1167-1170.

Mellors, J.W., A. Munoz, J.V. Giorgi, et al. 1997. Plasma viral load and CD4+ lymphocytes as prognostic markers of HIV-1 infection. Annals of Internal Medicine 126 (12): 946-954.

Moore, R.D., and R.E. Chaisson. 1999. Natural history of HIV infection in the era of combination antiretroviral therapy. AIDS 13 (14): 1933-1942.. PMID 10513653. https://doi. org/10.1097/00002030-199910010-00017.

Pharmacychecker. 2017. https://www.pharmacychecker.com/generic/price-comparison/ zidovudine/300+mg. Accessed 29 July 2017.

Rodger AJ et al. for the PARTNER Study Group. 2016. Sexual activity without condoms and risk of HIV transmission in serodifferent couples when the HIV-positive partner is using suppressive antiretroviral therapy. JAMA 316 (2): 1-11. https://doi.org/10.1001/jama.2016.5148. http:// jama.jamanetwork.com/article.aspx?doi=10.1001/jama.2016.5148. Accessed 12 July 2016.

Rodger AJ, V. Cambiano, T. Bruun, et al. 2018. Risk of HIV transmission through condomless sex in MSM couples with suppressive ART: The PARTNER2 Study extended results in gay men. In 22nd International AIDS conference (AIDS 2018), Amsterdam, the Netherlands. Oral Abstract WEAX0104LB.

Rosen, S., and M.P. Fox. 2011. Retention in HIV care between testing and treatment in SubSaharan Africa: A systematic review. PLOS Medicine 8 (7): e1001056.

Rowley, C.F., I.J. MacLeod, D. Maruapula, B. Lekoko, S. Gaseitsiwe, M. Mine, et al. 2016. Sharp increase in rates of HIV transmitted drug resistance at antenatal clinics in Botswana demonstrates the need for routine surveillance. Journal of Antimicrobial Chemotherapy 71 (5): $1361-1366$.

Samuel, R., M.N. Julian, R. Paredes, R. Parboosing, P. Moodley, L. Singh, et al. 2016. HIV-1 drug resistance by ultra-deep sequencing following short course zidovudine, single-dose nevirapine, and single-dose tenofovir with emtricitabine for prevention of mother-to-child transmission. JAIDS Journal of Acquired Immune Deficiency Syndromes 73 (4): 384-389.

Severe, Patrice, Marc Antoine Jean Juste, Alex Ambroise, Ludger Eliacin, Claudel Marchand, Sandra Apollon, Alison Edwards, Heejung Bang, Janet Nicotera, Catherine Godfrey, Roy M. Gulick, Warren D. Johnson Jr., Jean William Pape, and Daniel W. Fitzgerald. 2010. Early versus standard antiretroviral therapy for HIV-infected adults in Haiti. New England Journal of Medicine 363 (3): 257-265. https://doi.org/10.1056/NEJMoa0910370.

Smart, T. 2006. PEPFAR and Global Fund both highly effective, but is the funding sustainable? AIDSMAP. http://www.aidsmap.com/PEPFAR-and-Global-Fund-both-highly-effective-butis-the-funding-sustainable/page/1424121. Accessed 23 June 2006.

Strategies for Management of Antiretroviral Therapy Study Group, Sean Emery, Jacqueline A. Neuhaus, Andrew N. Phillips, Abdel Babiker, Calvin J. Cohen, Jose M. Gatell, Pierre-Marie Girard, Birgit Grund, Matthew Law, Marcelo H. Losso, Adrian Palfreeman, and Robin Wood. 2008. Major clinical outcomes in antiretroviral therapy (ART)-naive participants and in those not receiving ART at baseline in the SMART study. The Journal of Infectious Diseases 197 (8): 1133-1144. https://doi.org/10.1086/586713.

Stringer, J.S.A., M.S. McConnell, J. Kiarie, O. Bolu, T. Anekthananon, T. Jariyasethpong, et al. 2010. Effectiveness of non-nucleoside reverse-transcriptase inhibitor-based antiretroviral therapy in women previously exposed to a single intrapartum dose of nevirapine: A multi-country, prospective cohort study. PLOS Medicine 7 (2): e1000233.

Taylor-Smith, K., H. Tweya, A. Harries, E. Schoutene, and A. Jahn. 2010. Gender differences in retention and survival on antiretroviral therapy of HIV-1 infected adults in Malawi. Malawi Medical Journal: The Journal of Medical Association of Malawi 22 (2): 49-56.

Tenthani, Lyson, Andreas D. Haas, Hannock Tweya, Andreas Jahn, Joep J. van Oosterhout, Frank Chimbwandira, Zengani Chirwa, Wingston Ng'Ambi, Alan Bakali, Sam Phiri, Landon Myer, Fabio Valeri, Marcel Zwahlen, Gilles Wandeler, and Olivia Keiser. 2014. Retention in care under universal antiretroviral therapy for HIV infected pregnant and breastfeeding women ("Option B+”) in Malawi. AIDS 28 (4): 589-598. https://doi.org/10.1097/QAD.0000000000000143. 
The INSIGHT START Study Group. 2015. Initiation of antiretroviral therapy in early asymptomatic HIV infection. New England Journal of Medicine 373 (9): 795-807. https://doi. org/10.1056/NEJMoa1506816.

UNAIDS. 2017. 90-90-90 - An ambitious treatment target to help end the aids epidemic. http:// www.unaids.org/en/resources/documents/2017/90-90-90

Wade, N.A., G.S. Birkhead, B.L. Warren, T.T. Charbonneau, P.T. French, L. Wang, et al. 1998. Abbreviated regimens of zidovudine prophylaxis and perinatal transmission of the human immunodeficiency virus. New England Journal of Medicine 339 (20): 1409-1414.

Wilfert, C.M. 2003. HIVNET 012 and Petra. The Lancet 363 (9404): 244-245.

World Health Organization. 2003a. 3 by 5 Strategy: Making it happen. http://www.who.int/3by5/ publications/documents/en/3by5StrategyMakingItHappen.pdf

. 2003b. Scaling up antiretroviral therapy in resource-limited settings: Treatment guidelines for a public health approach. http://www.who.int/hiv/pub/prev_care/en/ARTrevision2003en. pdf?ua $=1$

. 2006. Antiretroviral therapy for HIV infection in adults and adolescents: Recommendations for a public health approach (2006 revision). http://www.who.int/hiv/pub/ART/adult/en

. 2009. Rapid advice: antiretroviral therapy for HIV infection in adults and adolescents. http://www.who.int/hiv/pub/arv/rapid_advice_art.pdf

2016. Global Action Plan on HIV Drug Resistance 2017-2021. https://www.who.int/hiv/ drugresistance/hivdr_darft_gap.pdf

2017. HIV drug resistance report 2017. Geneva: World Health Organization. http://apps. who.int/iris/bitstream/handle/10665/255896/9789241512831-eng.pdf;jsessionid=9AFD8 EB2096DEF42908467A854A90A9E?sequence=1

. 2018. WHO HIV data and statistics. http://www.who.int/hiv/data/en

Open Access This chapter is licensed under the terms of the Creative Commons Attribution 4.0 International License (http://creativecommons.org/licenses/by/4.0/), which permits use, sharing, adaptation, distribution and reproduction in any medium or format, as long as you give appropriate credit to the original author(s) and the source, provide a link to the Creative Commons licence and indicate if changes were made.

The images or other third party material in this chapter are included in the chapter's Creative Commons licence, unless indicated otherwise in a credit line to the material. If material is not included in the chapter's Creative Commons licence and your intended use is not permitted by statutory regulation or exceeds the permitted use, you will need to obtain permission directly from the copyright holder.

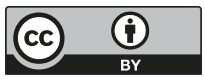

